Methods Longitudinal data from the annual British Household Panel Survey and Understanding Society (1994-2016) were examined with discrete-time event history analyses. 14992 youths contributed data for up to five observations (67 556 person-years) representing ages 11-15 years, with data rightcensored at the year of smoking initiation (or age 15). We examined associations between smoking ban implementation (2006 for Scotland, 2007 for other UK countries) and youth smoking initiation (i.e. trying smoking for the first time), before and after adjustment for parental smoking, and tested for interactions between parental smoking and the ban implementation. Multiple imputation was used to handle missing data. Analyses were adjusted for youth sex, age, UK country, socioeconomic status, and temporal trends in initiation.

Results Youth initiation of smoking was less likely after the implementation of the smoke-free legislation than before (OR: 0.16 ; $95 \%$ CI 0.14 to 0.18 ), and this difference further increased with each year after implementation (OR per year after the ban:0.88; 95\% CI 0.82 to 0.94). Adjustment for parental smoking only marginally attenuated the association with ban implementation (OR: 0.20 ; 95\% CI 0.16 to 0.24 ) and the per-year decrease after the ban (OR: 0.86 ; $95 \% \mathrm{CI}$ 0.80 to 0.92 ) was similar. There was an interaction between the ban implementation and parental smoking ( $p$-value: 0.001) such that parental smoking was more strongly associated with youth smoking initiation before the ban (OR: 1.41 ; 95\% CI 1.26 to 1.58 ) than after (OR: $0.92 ; 95 \%$ CI 0.65 to 1.32 ).

Discussion Changes in parental smoking behaviour did not seem to be a major explanation for the strong impacts of smoke-free legislation on youth smoking initiation, suggesting other mechanisms may be at work. The influence of parental smoking on youth initiation weakened after the ban which may indicate the displacement of parental smoking behaviour out of the home.

\section{OP58 ASSOCIATIONS BETWEEN PARENTAL VAPING AND YOUTH SMOKING AND VAPING IN THE UK: CROSS- SECTIONAL SURVEY FINDINGS}

${ }^{1} \mathrm{MJ}$ Green*, ${ }^{1} \mathrm{~L}$ Gray, ${ }^{1} \mathrm{H}$ Sweeting, ${ }^{2} \mathrm{M}$ Benzeval. ${ }^{1} \mathrm{MRC/CSO}$ Social and Public Health Sciences Unit, University of Glasgow, Glasgow, UK; ${ }^{2}$ Institute for Social and Economic Research, University of Essex, Colchester, UK

\subsection{6/jech-2018-SSMabstracts.57}

Background E-cigarette use has been rising steadily in the UK since 2011. Mounting evidence suggests e-cigarette use ('vaping') is safer than traditional cigarettes ('smoking'), and can aid smoking cessation. Nevertheless concerns are still raised that vaping may re-normalise smoking behaviour and help introduce youth to traditional cigarettes. Parental smoking is long-established as a risk factor for youth smoking, so parental vaping may be an important component of any normalisation effects of e-cigarettes on youth. We estimated associations of parental vaping with youth smoking and vaping.

Methods Data were from the 7th wave of the Understanding Society study, a representative annual panel study of UK households. Youth and parents self-reported current vaping and current and past smoking (parental vaping/smoking coded for any use in couples). Logistic regression analyses were conducted in STATA on 2373 youth aged 10-15 with valid data
(65\% of 3635 interviewed; 51\% female). Models were weighted for study non-response and adjusted for potential confounders (gender, age, UK country, ethnic minority, single parent status, parental education, occupation, income and parental smoking). Where there were interactions between parental vaping and parental smoking (never, ex or current), we estimated ORs within strata of parental smoking. With responses from previous surveys we additionally examined current smoking in youth who had never smoked before $(n=2,243$; i.e. initiation of smoking).

Results Vaping and smoking were rare among youth $(3.5 \%$ and $2.3 \%$ respectively) but more youth had parents who vaped $(11.6 \%)$ or were ex $(39.7 \%)$ or current $(24.9 \%)$ smokers. Parental vaping was not clearly associated with youth vaping after adjustment for confounders (OR: 1.86; 95\% CI 0.70 to 4.96). However, associations between parent and youth vaping varied by parental smoking ( $p$-value for interaction: 0.043 ) being strongest among youth whose parents were exsmokers (OR: 4.99; 95\% CI 0.94 to 26.53). After adjustment, parental vaping was not clearly associated with youth current smoking either (OR: 1.26 ; 95\% CI 0.38 to 4.19), which held across strata of parental smoking (interaction p-value: 0.102). However, parental vaping was associated with youth smoking initiation, even after adjustment (OR: 4.63; 95\% CI 1.29 to 16.58 ), and this too did not vary by parental smoking status (interaction p-value: 0.115 ).

Conclusion There was not strong evidence that parental vaping encouraged youth smoking or vaping overall. However, some indications of increased risk (e.g. for smoking initiation, or among youth whose parents were ex-smokers) warrant further study and monitoring to further inform regulation, especially as increasing numbers of smokers (including parents) switch to e-cigarettes.

\section{OP59 \#HAVE E-CIGARETTES RE-NORMALIZED OR DISPLACED YOUTH SMOKING?: A SEGMENTED REGRESSION ANALYSIS OF REPEATED CROSS SECTIONAL SURVEY DATA IN ENGLAND, SCOTLAND AND WALES}

${ }^{1}$ B Hallingberg*, ${ }^{2} \mathrm{O}$ Maynard, ${ }^{3} \mathrm{~L}$ Gray, ${ }^{4} \mathrm{~A}$ MacKintosh, ${ }^{1} \mathrm{E}$ Lowthian, ${ }^{1} \mathrm{G}$ Moore. ${ }^{1}$ School of Social Science, Cardiff University, Cardiff, UK; ${ }^{2}$ School of Experimental Psychology, University of Bristol, Bristol, UK; ${ }^{3} \mathrm{MRC} / \mathrm{CSO}$ Social and Public Health Sciences Unit, University of Glasgow, Glasgow, UK; ${ }^{4}$ Institute for Social Marketing, University of Stirling, Stirling, UK

\subsection{6/jech-2018-SSMabstracts.58}

Background Small yet significant impacts of e-cigarettes on population smoking cessations rates indicate promise for harm reduction. However, non-smoking young people are increasingly experimenting with e-cigarettes, and while regular use remains rare, arguments for regulation have been driven by fears that e-cigarettes re-normalize smoking. Others counter that e-cigarettes may displace youth smoking and further denormalize it. This study tests whether the secular decline in youth smoking prevalence, as well as perceived smoking norms, slowed or accelerated during the period from 20112015 (when e-cigarettes were emerging but prior to recent moves to regulate their use).

Methods Data were taken from the Smoking Drinking and Drug Use survey in England, Health Behaviour in School Aged Children/School Health Research Network surveys in 